\title{
类石墨烯碳材料的制备及其电容性能研究
}

\author{
苏善金来庆学梁彦瑜* \\ (南京航空航天大学材料科学与技术学院 南京 210016)
}

\begin{abstract}
摘要 利用三聚氰胺高温下缩聚产生的 $\mathrm{g}-\mathrm{C}_{3} \mathrm{~N}_{4}$ 作为二维模板, 同时利用其与对苯二甲醛发生席夫碱反应产生聚合物以 及聚合物的碳化, 制备了具有二维形貌的类石墨烯片层碳材料(GLCS), 随后, 将 GLCS 用 KOH 进行活化, 得到了具有 多孔结构的 a-GLCS. 对材料进行透射电镜(TEM)、扫描电镜(SEM)、X 射线光电子能谱测试(XPS)、拉曼光谱(Raman) 和比表面积分析(BET), 并将材料用作超级电容器电极材料进行电化学测试. 结果表明该方法能够制备得到二维片层 碳材料, 并且材料中掺杂有一定量的氮元素. GLCS 经过 KOH 活化后, 比表面积和电容性能都有了大幅提高, 电流密度 为 $1 \mathrm{~A} / \mathrm{g}$ 时 GLCS 和 a-GLCS 比容量分别为 160 和 $300 \mathrm{~F} / \mathrm{g}$.
\end{abstract}

关键词 二维模板; 席夫碱反应; 石墨烯; 活化; 超级电容器

\section{Synthesis of Graphene-like Carbon Sheets (GLCS) and Its Supercapacitor Property}

\author{
Su, Shanjin Lai, Qingxue Liang, Yanyu* \\ (College of Material Science and Engineering, Nanjing University of Aeronautics and Astronautics, Nanjing 210016)
}

\begin{abstract}
As a new kind of carbon material, graphene has unique two-dimensional (2D) structure and outstanding physicochemical properties, such as high electrical conductivity and large surface area $\left(2675 \mathrm{~m}^{2} / \mathrm{g}\right)$. In recent years, graphene has exhibited great potential for application as electrode materials in supercapacitors. In this work, a layered graphic carbon nitride $\left(\mathrm{g}-\mathrm{C}_{3} \mathrm{~N}_{4}\right)$ which was generated through polycondensation of melamine was employed as a $2 \mathrm{D}$ self-sacrificing template. Then, a nitrogen-doped graphene-like 2D carbon sheets (GLCS) was prepared by the calcination of melamine and terephthalaldehyde. This graphene synthesis method is a more facile and cost-effective route comparing with traditional synthetic methods, such as chemical exfoliation of graphite and chemical vapor deposition (CVD). In a typical synthesis, melamine was used as precursor of template and reactant of Schiff-base reaction. The $g-\mathrm{C}_{3} \mathrm{~N}_{4}$ template was used to confine the as-formed polymer to the interlayer gaps at about $600{ }^{\circ} \mathrm{C}$. Then, as the temperature continued to rise, the g- $\mathrm{C}_{3} \mathrm{~N}_{4}$ template undergoes complete thermolysis and the polymer between interlayer gaps was carbonized to be GLCS. Then, after activated by $\mathrm{KOH}$, a porous a-GLCS with high specific surface area and pore volume was obtained. GLCS and a-GLCS were characterized by transmission electron microscopy (TEM), scanning electron microscopy (SEM), X-ray photoelectron spectroscopy (XPS), Brunauer-Emmett-Teller (BET) surface area measurement, Raman spectroscopy etc. TEM and SEM results showed that GLCS showed well-defined two-dimensional structure, XPS analysis determined the nitrogen-containing functional groups present on the surface of the sample, BET surface area measurement showed that after activated by $\mathrm{KOH}$, the surface area have been obviously improved from $262.8 \mathrm{~m}^{2} / \mathrm{g}$ to $478.5 \mathrm{~m}^{2} / \mathrm{g}$. The electrochemical measurement showed that, at the current density of $1 \mathrm{~A} / \mathrm{g}$, the specific capacitance of GLCS and a-GLCS are 150 and $300 \mathrm{~F} / \mathrm{g}$ respectively. When the current density increased up to $20 \mathrm{~A} / \mathrm{g}$ the specific capacitance remained 100 and $200 \mathrm{~F} / \mathrm{g}$ respectively. The increasing of the specific capacitance was considered to be due to the generation of microporous during the activation process.
\end{abstract}

Keywords template; Schiff-base reaction; graphene; activated; supercapacitors

\section{1 引言}

全球经济的快速发展造成了化石燃料的过度消耗, 引起了一系列环境的破坏和污染问题, 威胁着人类的生 存和可持续发展, 需要开发高效、环保、可持续的能量 转换和存储装置来替代传统装置. 在众多新型储能装置 当中, 超级电容器兼具了电池和传统的静电电容器的优
点，具有高功率密度、较高的能量密度、充放电时间短、 使用寿命长和工作温度范围宽的特点, 成为新能源储能 装置领域的热点 ${ }^{[1 \sim 4]}$. 但是, 相对低的能量密度依然限 制着超级电容器的实际应用. 电极材料是超级电容器的 核心组成部件, 也是决定其性能的主要因素, 制备高比 容量碳材料对发展兼具高能量密度和高功率密度特性

*E-mail: liangyy403@126.com

Received March 25, 2015; published May 27, 2015.

Supporting information for this article is available free of charge via the Internet at http://sioc-journal.cn.

Project supported by the National Natural Science Foundation of China (Grant No. 21273114) and the Natural Science Foundation of Jiangsu Province (Grant No. BK2012791).

项目受国家自然科学基金面上(No. 21273114)和江苏省自然科学基金面上(No. BK2012791)资助. 
的新型超电容器具有重要意义 ${ }^{[5 \sim 9]}$.

石墨烯是由单层碳原子堆积形成的蜂窝状二维纳 米材料, 具有高理论比表面积 $\left(2675 \mathrm{~m}^{2} / \mathrm{g}\right)$ 、高导电性、 高电子迁移率、高稳定性等良好的物理化学性质, 使其 在能量储存与转化、催化、传感器以及功能性复合材料

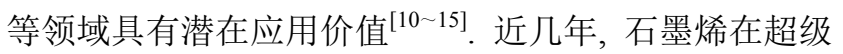
电容器电极材料领域的研究受到广泛关注. 2008 年, Ruoff 等 ${ }^{[16]}$ 用化学法还原氧化石墨烯(GO), 第一次将化 学修饰石墨烯材料用于超级电容器电极材料, 石墨烯比 表面积达 $705 \mathrm{~m}^{2} / \mathrm{g}$, 在水系和有机系电解液中的比容量 分别为 135 和 $99 \mathrm{~F} / \mathrm{g}$. Shi 等 ${ }^{[17]}$ 通过水热法加无水肼还原 $\mathrm{GO}$ 得到了具有三维交联结构的石墨烯水凝胶, 在 1 $\mathrm{mol} / \mathrm{L} \mathrm{H}_{2} \mathrm{SO}_{4}$ 中电流密度 $1 \mathrm{~A} / \mathrm{g}$ 时电容达 $220 \mathrm{~F} / \mathrm{g}$ 并具有 良好的倍率性能和循环稳定性. 研究发现将 $\mathrm{GO}$ 还原后 得到的石墨烯其比表面积远小于理论值 $\left(2675 \mathrm{~m}^{2} / \mathrm{g}\right)$, 其 比容量也小于石墨烯的理论比容量(约 $500 \mathrm{~F} / \mathrm{g}$ ). 研究发 现 GO 经过化学法还原后含氧官能团含量降低, 从而减 小石墨烯片层之间的排斥力导致石墨烯片层再次团聚, 使得石墨烯有效比表面积下降, 阻碍电解质与石墨烯片 层表面结合从而降低其电化学活性. 为了解决石墨烯片 层团聚的问题, 许多研究者在石墨烯片层间引入支撑物 质来减少石墨烯片层堆叠, 或者在石墨烯之间产生孔道 来增加石墨烯与电解液的接触面积以及通过化学活化 提高材料的比表面积. 2011 年, Ruoff 等 ${ }^{[18]}$ 用 $\mathrm{KOH}$ 对石 墨烯进行活化得到了比表面积 $3100 \mathrm{~m}^{2} \cdot \mathrm{g}^{-1}$ 的活化石墨 烯. 在 $\mathrm{KOH}$ 的活化作用下石墨烯中生成大量孔径小于 1 $\mathrm{nm}$ 的微孔和小于 $50 \mathrm{~nm}$ 的介孔. 将其作为超级电容器 电极材料测试, 发现在 $500 \mathrm{mV} / \mathrm{s}$ 扫速下电容值达 150 $\mathrm{F} / \mathrm{g}$.

目前, 石墨烯的合成方法主要有机械剥离法、氧化-

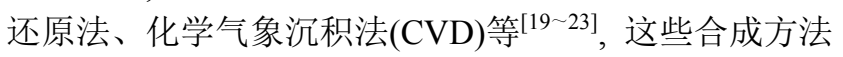
过程繁琐, 成本高, 不利于石墨烯材料的大量制备和应 用. $\mathrm{Li}$ 等 ${ }^{[24,25]}$ 开发出一种利用二维模板 $\mathrm{g}-\mathrm{C}_{3} \mathrm{~N}_{4}$ 制备石墨 烯碳材料的方法, 即通过双氰胺缩聚产生二维片层的 $\mathrm{g}-\mathrm{C}_{3} \mathrm{~N}_{4}$ 交联结构, 在二维片层间通过葡萄糖等小分子缩 聚形成二维碳片, 最后通过高温时 $\mathrm{g}-\mathrm{C}_{3} \mathrm{~N}_{4}$ 自动分解这 一特性, 最终得到了二维的类石墨烯碳材料.

我们利用三聚氰胺高温下缩聚产生的 $\mathrm{g}-\mathrm{C}_{3} \mathrm{~N}_{4}$ 作为 二维模板, 通过席夫碱反应制备得到了掺氮的类石墨烯 碳材料. 该方法不但快速简便的制备了石墨烯, 还实现 了对石墨烯的原位氮掺杂. 研究表明, 化学活化法是提 高碳材料比表面积改善其电容性能最常用到的方法, 其 中最常用的活化剂为 $\mathrm{KOH}^{[26 ~ 30]}$. $\mathrm{KOH}$ 活化能够有效的 在碳材料中引入孔道结构, 显著提高碳材料的比表面积 和电容性能. 此外, $\mathrm{KOH}$ 活化还具有活化温度低、产量 高等优点. 其缺点是对碳材料的结构的破坏, 一定程度 上降低了材料的导电性和含氮量以及其具有腐蚀性和 后续要水洗残留在碳材料中的试剂. 因此, 我们对合成
的材料进行了 $\mathrm{KOH}$ 活化, 通过在材料中产生孔道结构 来进一步提高材料的比表面积和电容性能.

\section{2 结果与讨论}

\section{1 材料制备}

a-GLCS 的制备过程如图 1 所示. 首先, 称取一定比 例的对苯二甲醛和三聚氰胺充分研磨使二者混合, 其中 三聚氰胺的量要远高于对苯二甲醛，原因是三聚氰胺不 仅是反应物前驱体, 还是二维模板 $\mathrm{g}-\mathrm{C}_{3} \mathrm{~N}_{4}$ 的来源. 然 后, 对混合物进行热处理, 热处理过程由两部分组成, 为模板的稳定形成 (600 ${ }^{\circ} \mathrm{C}$ 左右) 和模板的完全分解(高 于 $\left.700{ }^{\circ} \mathrm{C}\right)$. 在这一过程中, 三聚氰胺和对苯二甲醛发 生聚合反应生成聚合物(图 S1), 随着温度的继续升高, 聚合物碳化, 形成碳材料. 二维模板高温分解后, 模板 中间的类石墨烯片层材料(GLCS)便合成了. 最后, 将 GLCS 用 $\mathrm{KOH}$ 进行化学活化, 便得到了具有多孔结构 的二维类石墨烯碳材料.

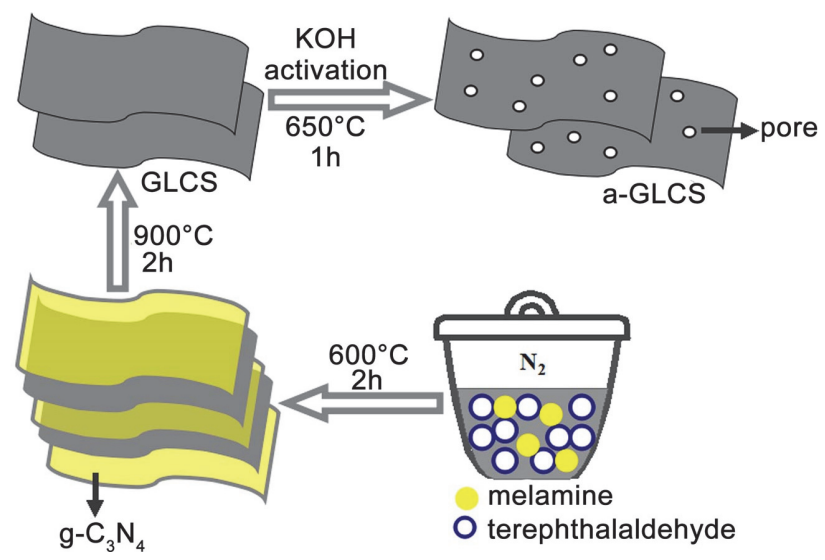

图 1 GLCS 和 a-GLCS 制备过程

Figure 1 Procedures for the preparation of GLCS and a-GLCS

\section{2 形貌和结构表征}

采用比表面积和孔径测定、拉曼(Raman)、扫描电 镜(SEM) 和透射电镜(TEM)对复合材料的形貌和结构进 行表征. 图 2(a,b)为 a-GLCS 的 TEM 图, 从图 2(a)可以 看到大范围的 a-GLCS 呈现出蓬松和有波纹的二维片层 结构形态, 图 2(b)高倍率下的 TEM 照片, 可以看到材料 局部表面有许多褶皱和卷曲的薄纱状片层. 图 2(c, d) 为 a-GLCS 的 SEM 图, 从图 2 中可以清楚地看到材料呈现 薄纱状, 表面有凸起的褶皱. TEM 图和 SEM 图的表征结 果说明该法合成的碳材料具有类似石墨烯的二维片层 形貌. 图 S2 给出了三聚氭胺与对苯二甲醛质量比为 1 : 1 时合成的材料的 TEM 和 SEM 表征图, 从图 S2 中可以 看出, 材料呈现因研磨造成的较厚的块状形貌, 原因是 在材料形成过程中没有形成足量的 $\mathrm{g}-\mathrm{C}_{3} \mathrm{~N}_{4}$ 模板来调控 材料的形貌, 说明了二维模板在调控材料形貌上的重要 作用. 


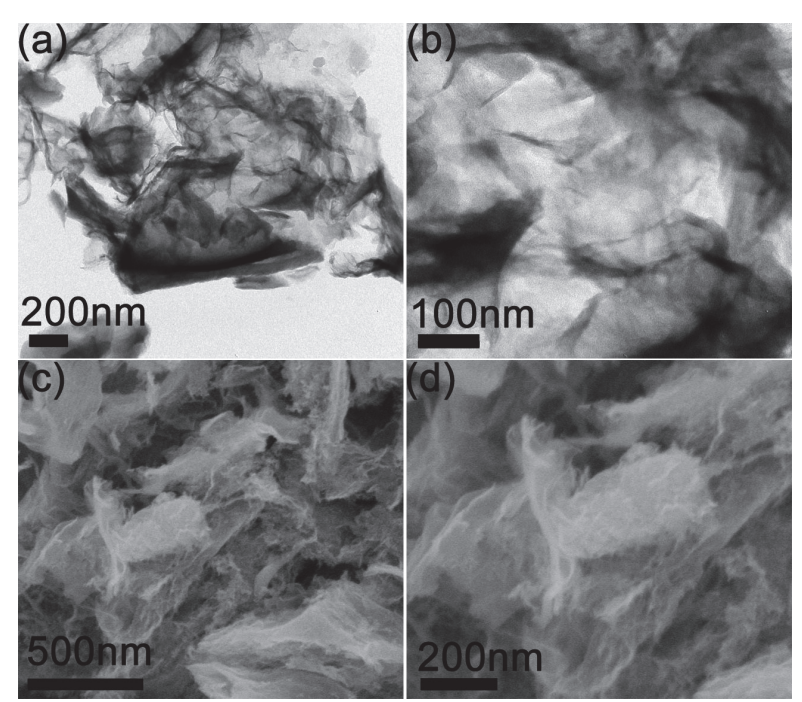

图 2 a-GLCS 的 TEM 图(a, b)和 SEM 图(c, d)

Figure 2 TEM images of a-GLCS $(a, b)$ and SEM images of a-GLCS (c, d)

图 3 是 GLCS 和 a-GLCS 的 Raman 光图谱, 从图 3 中可以观察到二者的 Raman 光谱图在 $1587 \mathrm{~cm}^{-1}$ 处展示 出一个宽泛蓝移的 $\mathrm{G}$ 峰, 该峰为石墨化的碳原子 $\left(\mathrm{sp}^{2}\right.$ 杂 化, 包括 $\mathrm{C}-\mathrm{C}, \mathrm{N}-\mathrm{C}$ ) 的切向伸缩振动; 在 $1362 \mathrm{~cm}^{-1}$ 处 展示出一个宽泛蓝移的 D 峰, 代表无序的和缺陷的碳 ( $\mathrm{sp}^{3}$ 杂化). $\mathrm{D}$ 峰和 $\mathrm{G}$ 峰处的峰强比 $\left(I_{\mathrm{D}} / I_{\mathrm{G}}\right)$ 是说明碳材料 石墨化程度的重要参数, 其中 $I_{\mathrm{D}} / I_{\mathrm{G}}$ 越小说明碳材料中 $\mathrm{sp}^{3}$ 的碳原子越少而 $\mathrm{sp}^{2}$ 的碳原子越多, 材料的石墨化程 度越高, 导电性越好; 而 $I_{\mathrm{D}} / I_{\mathrm{G}}$ 越大表明碳材料石墨化程 度越低或者缺陷越多, 经过计算 GLCS 和 a-GLCS 的 $I_{\mathrm{D}} / I_{\mathrm{G}}$ 分别为 0.90 和 0.96 , 说明 $\mathrm{KOH}$ 活化后, 材料的缺 陷结构增多, 破坏了部分 $\pi$ 电子共轭结构导致石墨化程 度降低.

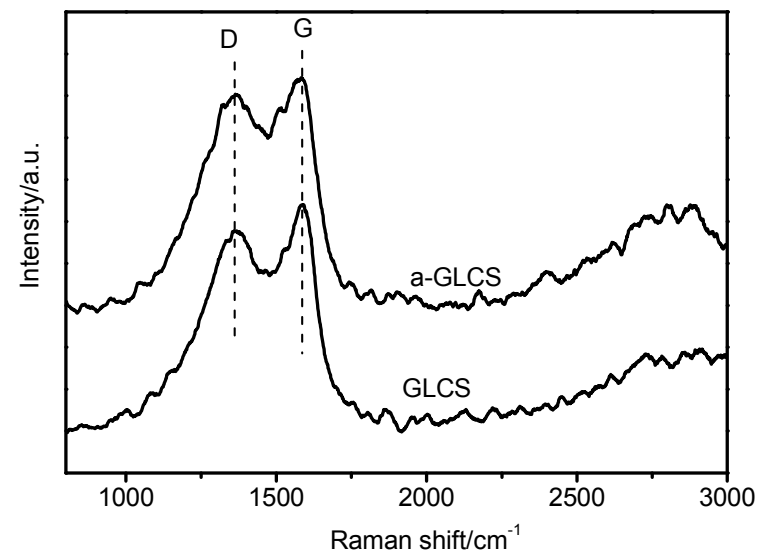

图 3 GLCS 和 a-GLCS 的 Raman 光谱图

Figure 3 The Raman spectra of GLCS and a-GLCS

图 4 为 a-GLCS 样品高分辨 N1s 谱图, 我们可以将 $\mathrm{N} 1 \mathrm{~s}$ 峰在 $398.2 \pm 0.1 、 400.0 \pm 0.1$ 和 $401.1 \pm 0.1 \mathrm{eV}$ 处拟
合出 3 个不同的峰，分别对应吡啶型氮、吡咯型氮和石 墨化型氮. XPS 测试结果表明, 样品 a-GLCS 中氮元素含 量为 $5.82 \%$. 当用作超级电容器电极材料, 掺杂的氮元 素起到的作用主要为增加材料导电性、提高材料亲水性 和通过产生噟电容来提高材料比容量 ${ }^{[31 \sim 35]}$. 相比对石 墨烯进行后续氮化处理进行掺氮来提高其电化学性能, 原位掺杂具有成本低和掺杂量高的优点.

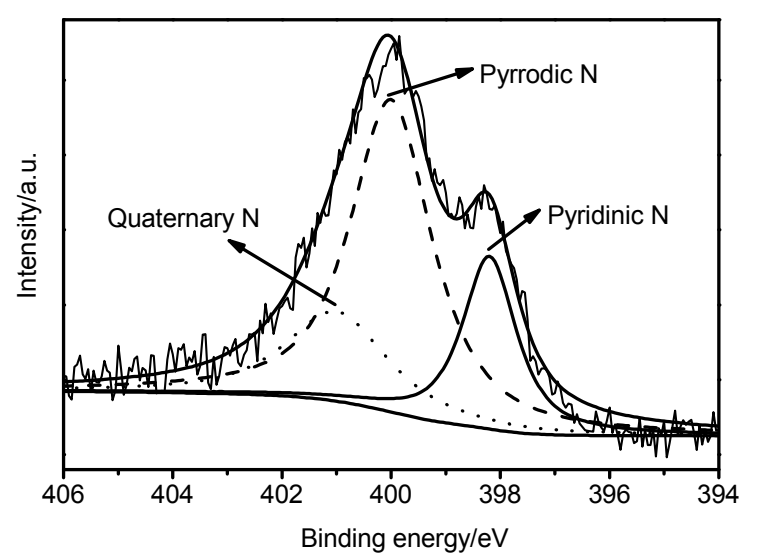

图 4 a-GLCS 的 XPS 测试 N1s 图

Figure 4 N1s XPS spectra of a-GLCS

图 5(a)为 GLCS 氮气吸脱附曲线, 图 5(b)为对应的 孔径分布图. 表征结果显示 GLCS 的比表面积为 262.8 $\mathrm{m}^{2} / \mathrm{g}$, 孔体积为 $1.16 \mathrm{~cm}^{3} / \mathrm{g}$. 从图 5 中可以清楚看出, 材 料中的孔主要是 $2 \sim 10 \mathrm{~nm}$ 的介孔和部分大孔. 图 5(c) 为 a-GLCS 氮气吸脱附曲线, 图 5(d)为对应的孔径分布 图. 表征结果显示 a-GLCS 的比表面积为 $478.5 \mathrm{~m}^{2} / \mathrm{g}$, 孔 体积为 $3.24 \mathrm{~cm}^{3} / \mathrm{g}$. 从吸脱附曲线的形状可以看出, 该 吸脱附曲线为 Type I 和 Type IV 结合的类型, 在 $P / P_{0}<$ 0.1 的低压区，有明显的 $\mathrm{N}_{2}$ 吸附，表明材料中存在微 $孔^{[30,36,37]}$. 在 $P / P_{0}=0.4 \sim 0.95$, 曲线中有明显滞留环, 表明在 a-GLCS 中有介孔 ${ }^{[38,39]}$, 微孔的存在是由于 $\mathrm{KOH}$ 的活化作用产生的. BET 测试结果显示 $\mathrm{KOH}$ 活化后, 材 料的比表面积增加了一倍左右, 并且增加的比表面积主 要是由于 $\mathrm{KOH}$ 活化产生的微孔结构贡献的.

\section{3 超电容性能}

图 6 给出了 GLCS 和 a-GLCS 在 $6 \mathrm{~mol} / \mathrm{L} \mathrm{KOH}$ 溶液 中的循环伏安曲线对比图, 电压扫描窗口为 $-1 \sim 0 \mathrm{~V}$ (vs. SCE), 扫描速度为 $5 \mathrm{mV} / \mathrm{s}$. 首先可以很容易的看出, 样品的循环伏安曲线都接近矩形形状, 表明材料电容主 要来源于双电层电容. A-GLCS 的曲线面积大小与 GLCS 相比却明显增大, 说明其比容量更高, 而比容量 的增加是由 $\mathrm{KOH}$ 的活化引起的. 图 7 是 GLCS 和 a-GLCS 在电流密度均为 $1 \mathrm{~A} / \mathrm{g}$ 时的恒流充放电曲线对 比图, 从图 6 中可以看到样品的充电曲线和放电曲线随 

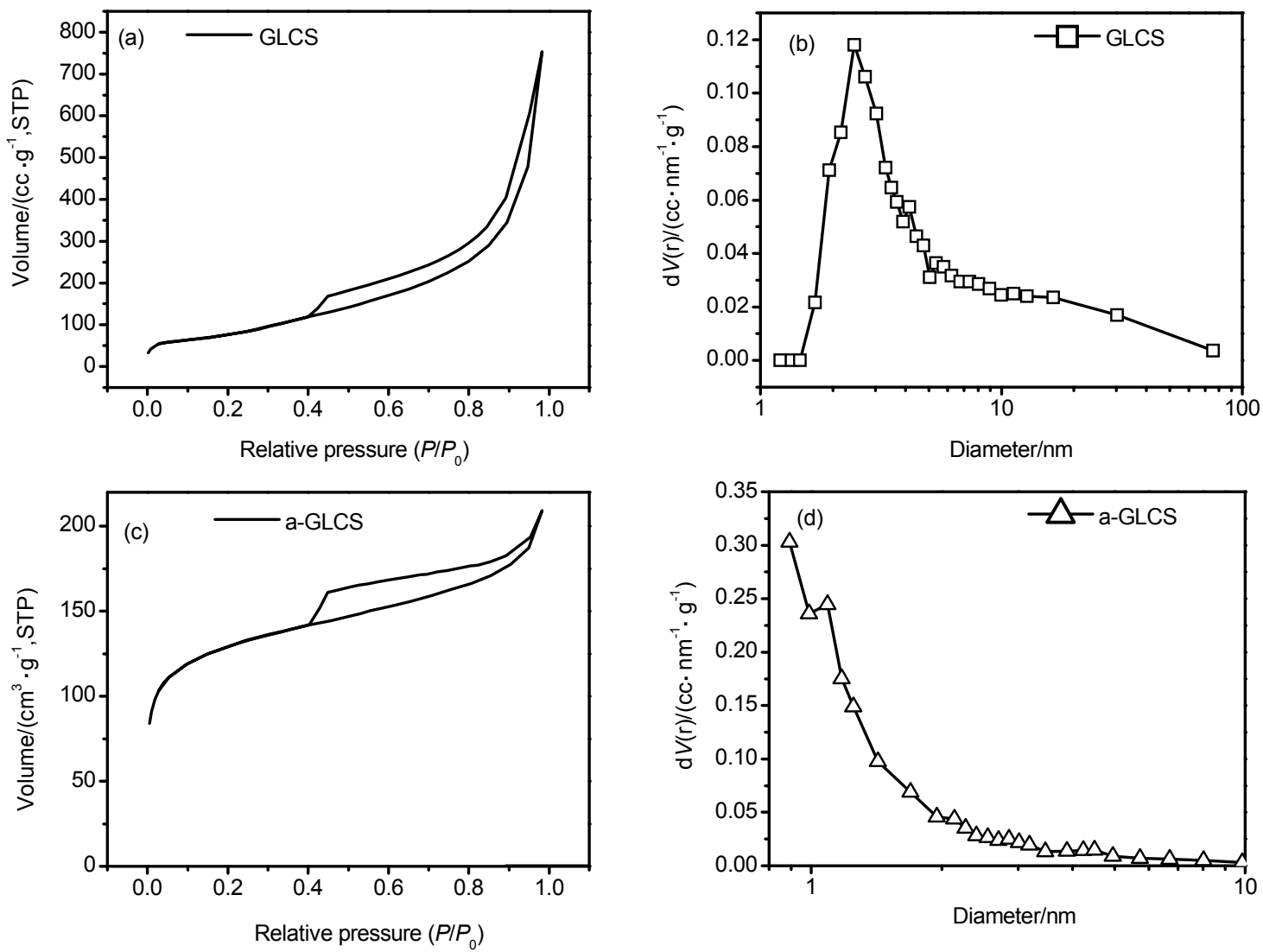

图 5 GLCS 和 a-GLCS 的 $(a, c)$ 氮气吸脱附曲线和 $(b, d)$ 对应的孔径分布图

Figure 5 Nitrogen adsorption-desorption isotherms (a, c) and pore-size distribution curve (b, d) of GLCS and a-GLCS

时间呈现线性变化, 充电时, 电极电压随充电时间的延 长呈递增趋势, 放电时, 电极电压随充电时间的延长呈 递减趋势，而且充电时间和放电时间基本相同，说明两 种电极材料具有良好的双电层电容性能而且可逆性良 好. 可以看到, a-GLCS 的一次充放电时间多于 GLCS, 说明其比容量更高, 这与循环伏安的测试结果一致.

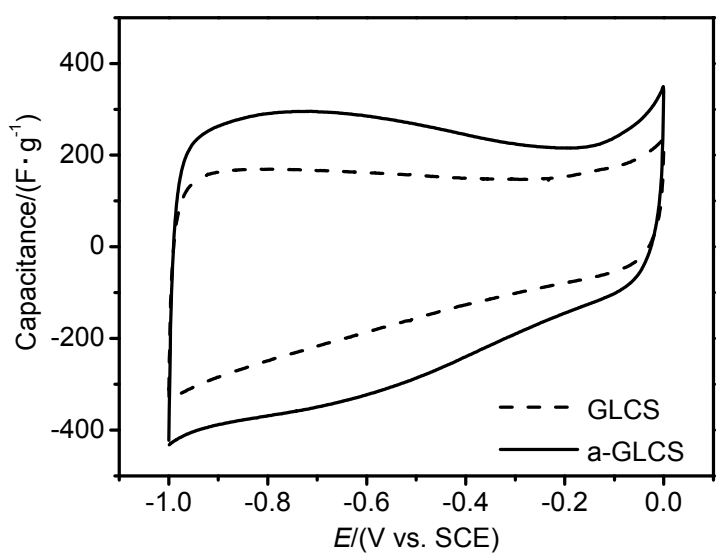

图 $6 \mathrm{GLCS}$ 和 a-GLCS 在 $6 \mathrm{~mol} / \mathrm{L} \mathrm{KOH}$ 溶液中扫速为 $5 \mathrm{mV} / \mathrm{s}$ 时的循 环伏安曲线

Figure $6 \mathrm{CV}$ curves of GLCS and a-GLCS at a scan rate of $5 \mathrm{mV} / \mathrm{s}$ in 6 $\mathrm{mol} / \mathrm{L} \mathrm{KOH}$

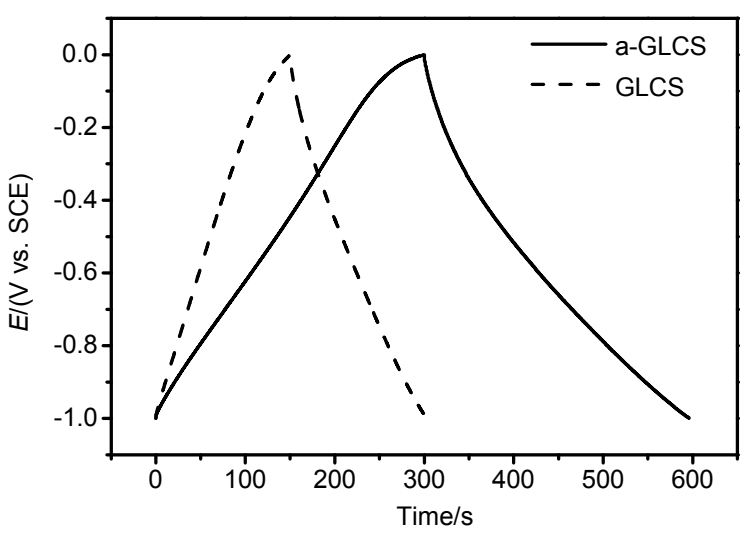

图 7 GLCS 和 a-GLCS 在电流密度 $1 \mathrm{~A} / \mathrm{g}$ 下的充放电测试曲线

Figure 7 Galvanostatic charge/discharge curves of GLCS and a-GLCS at current density of $1 \mathrm{~A} / \mathrm{g}$.

图 8 为 GLCS 和 a-GLCS 在不同电流密度下的比容 量曲线. 活化前 GLCS 的比容量在 $1 \mathrm{~A} / \mathrm{g}$ 时为 $175 \mathrm{~F} / \mathrm{g}$, 活化后增加到 $300 \mathrm{~F} / \mathrm{g} ; 20 \mathrm{~A} / \mathrm{g}$ 时比容量为 $100 \mathrm{~F} / \mathrm{g}$, 活化 后比容量为 $200 \mathrm{~F} / \mathrm{g}$. 以上结果显示, $\mathrm{KOH}$ 活化后, GLCS 比容量增加了一倍左右, a-GLCS 比容量的提高主要得 益于其经过 $\mathrm{KOH}$ 活化后形成的大量微孔结构. 前边比 
表面积分析结果显示 a-GLCS 的比表面积约为 GLCS 的 两倍, 说明材料的比容量在一定程度上跟材料的比表面 积成线性比例关系.

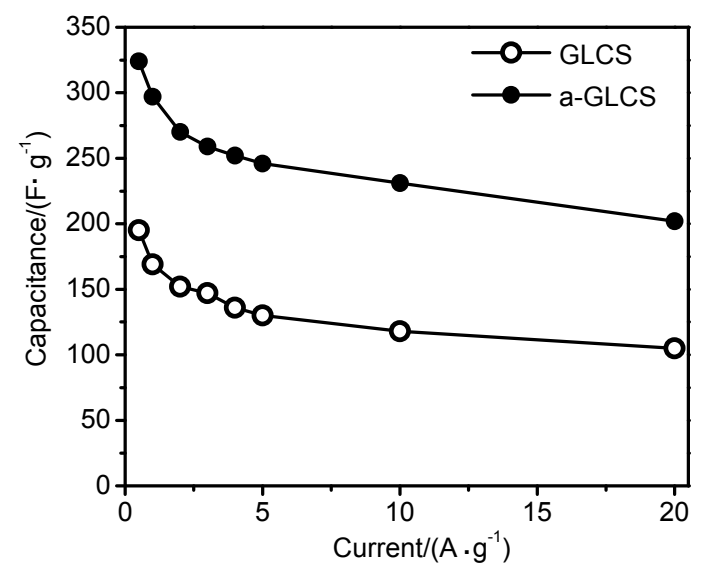

图 8 GLCS 和 a-GLCS 比容量和电流密度的关系

Figure 8 Specific capacitance versus different current density of GLCS and a-GLCS

为了获得更多电极材料内阻和电极/电解液阻抗的 信息, 我们对 GLCS 和 a-GLCS 进行电化学阻抗测试(图 9), 从测得的 Nyquist 阻抗图可以明显看出, 在高频区有 一个半圆形, 其中半圆直径代表电荷传输电阻 $\left(R_{\mathrm{ct}}\right)$, 半 圆直径越小则电荷传输电阻越小, 经计算 GLCS 和 $\mathrm{a}-\mathrm{GLCS}$ 的 $R_{\mathrm{ct}}$ 大小分别为 0.22 和 $0.26 \Omega$, 后者略大的原 因为, $\mathrm{KOH}$ 活化后造成材料中 $\mathrm{N}$ 元素减少以及石墨化程 度的降低, 增大了材料电阻. 在低频区, 曲线与横轴几 乎垂直表明材料具有优异的电容性能. 除此之外, 从高 频区中半圆与 $x$ 轴的交点可以得到两种材料内阻 $\left(R_{\Omega}\right)$ 均 为 $0.4 \Omega$, 该内阻主要由三部分组成, 即电极材料本身 的固有电阻、电解液内阻以及电极材料与集流体界面上 的电阻.

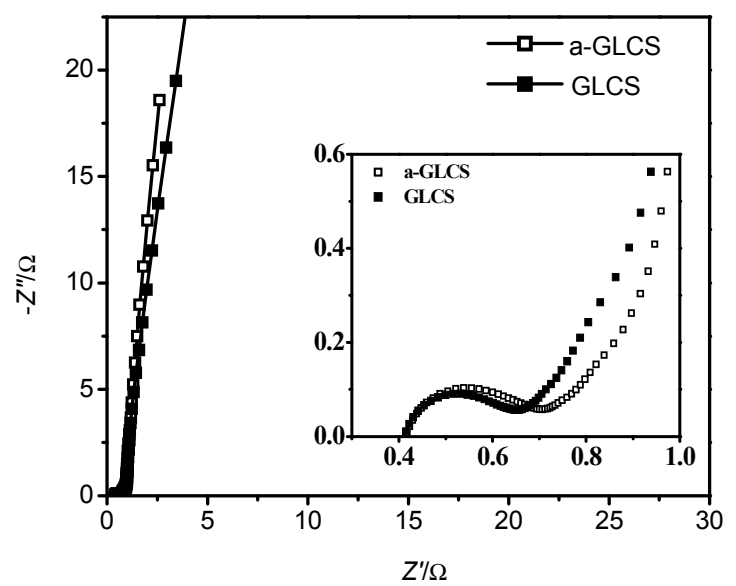

图 9 GLCS 和 a-GLCS 电化学交流阻抗测试(小图为高频区阻抗图) Figure 9 Nyquist plots of GLCS and a-GLCS The inset shows the spectra at high-frequency region
循环寿命是衡量超级电容器电化学特性的一个重 要指标，我们通过对 a-GLCS 电极材料进行充放电循环 稳定性测试来评估材料的稳定性, 在 $5 \mathrm{~A} / \mathrm{g}$ 的电流密度 下, $6 \mathrm{~mol} / \mathrm{L} \mathrm{KOH}$ 电解液中对 a-GLCS 电极材料进行 3000 次循环充放电测试, 循环性能曲线如图 10 所示. 从图 10 中可以看出, a-GLCS 电极材料在前 500 次的循 环中比容量出现一定程度的衰减, 500 次循环以后, 曲 线趋于平稳, 经过 3000 次充放电后电容仅损失 $10 \%$ 左 右，表现出了优异的循环稳定性.

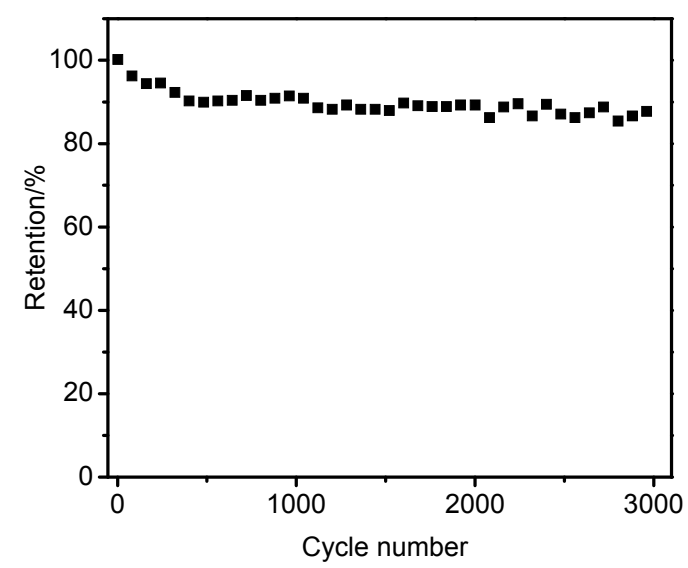

图 10 a-GLCS 在电流密度下为 $5 \mathrm{~A} / \mathrm{g}$ 下的循环寿命图

Figure 10 Cycling performance of a-GLCS electrode at a current density of $5 \mathrm{~A} / \mathrm{g}$

\section{3 结论}

本文利用 $\mathrm{g}-\mathrm{C}_{3} \mathrm{~N}_{4}$ 作为二维模板, 通过席夫碱反应 产生聚合物以及聚合物的后续碳化，制备了具有二维形 貌的类石墨烯片层碳材料(GLCS). 为了提高材料的电 容性能, 我们将 GLCS 用 $\mathrm{KOH}$ 进行化学活化, 得到了具 有多孔结构的 a-GLCS, 物性表征分析发现材料具有良 好的二维片层结构, 且掺杂有一定量的氮元素. 经过 $\mathrm{KOH}$ 活化后, 材料的比表面积有了明显提高, 同时电化 学性能也有了明显改善.

\section{4 实验部分}

\section{1 试剂与仪器}

三聚氰胺(CP, 国药集团化学试剂有限公司), 对苯 二甲醛 (AR, 上海达瑞精细化学品有限公司), 氢氧化钾 (AR, 南京化学试剂有限公司), 盐酸(AR, 南京化学试 剂有限公司), 无水乙醇(AR, 南京化学试剂有限公司), CHI660E 电化学工作站(上海辰华仪器公司).

\section{2 材料的合成}

称取 $200 \mathrm{mg}$ 对苯二甲醛和 $4 \mathrm{~g}$ 三聚氰胺置于玛瑙研 钵中研磨, 使两种反应物混合均匀, 然后将混合物转移 至 $10 \mathrm{~mL}$ 带盖的陶瓷坩埚中; 将陶瓷坩埚放入管式炉中 进行热处理, 热处理程序结束后, 待温度自然冷却至室 
温, 取出坩埚, 直接收集坩埚内的样品, GLCS 样品无需 后续处理可以直接使用; 将得到的 GLCS 用 $\mathrm{KOH}$ 溶液 浸润, 其中 GLCS 和 $\mathrm{KOH}$ 的质量比为 $1: 3$, 烘干并转 移至 $10 \mathrm{~mL}$ 带盖的陶瓷坩埚中; 将陶瓷坩埚放入管式炉 中进行活化热处理, 以 $5{ }^{\circ} \mathrm{C} / \mathrm{min}$ 升温速率升温至 $650{ }^{\circ} \mathrm{C}$, 保温 $1 \mathrm{~h}$; 热处理程序结束后, 待温度自然冷却 至室温, 取出坩埚, 取出样品并放入玛瑙研钵研磨成均 匀粉末, 用稀盐酸和蒸馏水清洗掉残留的 $\mathrm{KOH}$, 即得到 a-GLCS，干燥备用.

\section{3 材料的物性表征和电化学测试}

扫描电子显微镜(SEM)测试采用德国 LEO-1550 场 发射扫描电子显微镜; 透射电子显微镜(TEM)测试采用 荷兰 FEI 公司生产的 Tecnai G2 透射电镜; 激光拉曼光 谱测试(Raman)采用德国 Leica 公司生产的 TCS SP5 II 激光共聚焦显微镜(激发波长为 $532 \mathrm{~nm}$ ); 氮气吸/脱附 等温曲线、比表面积和孔径分布曲线采用美国 Micromeritics 公司 ASAP 2020 物理吸附仪于 $77 \mathrm{~K}$ 条件 下获得, 孔径分布依据 BJH 方法计算得到; $X$ 射线光电 子能谱测试(XPS)采用日本岛津-Kratos 公司生产的 AXIS UltraDLD X 射线光电子能谱仪.

电化学电容性能测试在 $\mathrm{CHI} 660 \mathrm{E}$ 电化学工作站上 进行, 电解液为 $6 \mathrm{~mol} / \mathrm{L} \mathrm{KOH}$ 溶液, 采用三电极体系, 其中对电极为铂电极, 参比电极为饱和甘录电极, 工作 电极的制备步骤如下: 按照质量比 $m$ (活性物质) $: m$ (乙 炔黑) $: m(\mathrm{PTEF})=8: 1: 1$ 的比例将三者在玛瑙研钵中 混合研磨，一般单个电极中活性物质的质量在 $5 \mathrm{mg}$ 左 右; 将研磨好的膏状混合物涂在预先处理好的泡沫镍集 流体上, 再将涂好的电极放在真空干燥箱中 $80{ }^{\circ} \mathrm{C}$ 下干 燥 $24 \mathrm{~h}$; 取出电极后在 $10 \mathrm{MPa}$ 压力下进行压片. 电极 材料的电容性能测试主要包括循环伏安测试和恒流充 放电测试.

\section{References}

[1] Lu, X.; Yu, M.; Wang, G. Energy Environ. Sci. 2014, 7, 2160.
[2] Hall, P. J.; Mirzaeian, M.; Fletcher, S. I.; Sillars, F. B. Energy Environ. Sci. 2010, 3, 1238.

[3] Wang, G.; Zhang, L.; Zhang, J. Chem. Soc. Rev. 2012, 41, 797.

[4] Wood, K. N.; O'hayre, R.; Pylypenko, S. Energy Environ. Sci. 2014, $7,1212$.

[5] Zhai, Y.; Dou, Y.; Zhao, D. Adv. Mater 2011, 23, 4828.

[6] Kim, H.; Fortunato, M. E.; Xu, H. J. Phys. Chem. C 2011, 115, 20481.

[7] Jiang, H.; Lee, P. S.; Li, C. Energy Environ. Sci. 2013, 6, 41.

[8] Dai, L.; Chang, D. W.; Baek, J. B. Small 2012, 8, 1130.

[9] Hu, H.; Hu, Z.; Ren, X. Chin. J. Chem. 2015, 33, 199

[10] Wen, L.; Liu, C.; Cheng, H. Acta Chim. Sinica 2014, 72, 333. (闻雷, 刘成名, 成会明, 化学学报, 2014, 72, 333.)

[11] Wang, Y.; Yu, X.; Huang, S.; Wang, Z. Acta Chim. Sinica 2014, 72, 378. (王跃，余旭丰，黄少铭，王志民，化学学报, 2014, 72, 378.)

[12] Chou, T. C.; Huang, C. H.; Doong, R. A. J. Mater. Chem. A 2013, 1, 2886.

[13] Sheng, Z. H.; Shao, L.; Chen, J. J. ACS Nano 2011, 5, 4350.

[14] Du, Q.; Zheng, M.; Zhang, L. Electrochim. Acta 2010, 55, 3897.

[15] Yu, X.; Sheng, K.; Shi, G. Acta Chim. Sinica 2014, 72, 319. (于小 雯, 盛凯旋, 石高全, 化学学报, 2014, 72,319.)

[16] Stoller, M. D.; Park, S. J.; Zhu, Y. W.; An, J. H.; Ruoff, R. S. Nano Lett. 2008, 8, 3498

[17] Xu, Y.; Sheng, K.; Li, C.; Shi, G. ACS Nano 2010, 4, 4324

[18] Zhu, Y.; Murali, S.; Stoller, M. D.; Ruoff, R. S. Science 2011, 332, 1537.

[19] Luo, B.; Liu, S.; Zhi, L. Small 2012, 8, 630.

[20] Fang, N.; Liu, F.; Miao, L.; Jiang, J. Acta Chim. Sinica 2012, 70, 2197. (方楠, 刘风，缪灵，江建军，化学学报, 2012, 70, 2197.)

[21] Feng, H.; Wu, Y.; Li, J. Small 2014, 10, 2233.

[22] Huang, L.; Wu, B.; Chen, J. Small 2013, 9, 1330.

[23] Chen, J.; Wu, B.; Liu, Y. Acta Chim. Sinica 2014, 72, 359. (陈集思, 武斌, 刘云圻, 化学学报, 2014, 72, 359.)

[24] Li, X. H.; Kurasch, S.; Kaiser, U. Angew. Chem., Int. Ed. 2012, 51 , 9689.

[25] Li, X. H.; Antonietti, M. Angew. Chem., Int. Ed. 2013, 52, 4572.

[26] Wang, J.; Kaskel, S. J. Mater. Chem. 2012, 22, 23710.

[27] Xiao, N.; Tan, H.; Zhu, J. ACS Appl. Mater. Interfaces 2013, 5, 9656.

[28] Xu, G.; Ding, B.; Nie, P. Chem. Eur. J. 2013, 19, 12306.

[29] Xu, Y.; Lin, Z.; Huang, X. Adv. Mater. 2013, 25, 5779.

[30] Zhou, M.; Pu, F.; Wang, Z. Carbon 2014, 68, 185.

[31] Chen, L. F.; Zhang, X. D.; Liang, H. W. ACS Nano 2012, 6, 7092.

[32] Hao, L.; Luo, B.; Li, X. Energy Environ. Sci. 2012, 5, 9747.

[33] Zhou, D. D.; Li, W. Y.; Dong, X. L. J. Mater. Chem. A 2013, 1 , 8488.

[34] Zhu, H.; Yin, J.; Wang, X. Adv. Funct. Mater. 2013, 23, 1305.

[35] Han, J.; Zhang, L. L.; Lee, S. ACS Nano 2012, 7, 19.

[36] Su, Y. S.; Arumugam, M. Nat. Commun. 2012, 3, 1166.

[37] Kruk, M.; Jaroniec, M. Chem. Mater. 2001, 13, 3169

[38] Prasad, Y.; Abhik, B.; Satishchandra, O. ChemSusChem 2012, 5, 2159.

[39] Li, Z.; Xu, Z.; Mitlin, D. Energy Environ. Sci. 2013, 6, 871.

(Qin, X.) 